\title{
Image Compression based on Scaling Functions and Wavelet Transformations
}

\author{
A. J. Rajeswari Joe \\ Research Scholar \\ Bharathiyar University \\ Assistant Professor, \\ GSS Jain College For Women, \\ Chennai
}

\author{
N.Rama, Ph.D \\ Associate Professor, \\ Presidency College
}

\begin{abstract}
The objective of this paper is to implement and evaluate the effectiveness of scaling functions and wavelet transformations in the field of image compression and decompression. The performance parameters like Peak Signal to Noise Ratio (PSNR), Mean Squared Error (MSE), and Compression Ratio(CR),SNR(Signal to Noise Ratio) are calculated based on the Matlab source code. The implemented model provides better PSNR, MSE, CR, SNR than the Basic 2D Discrete Cosine Transform.
\end{abstract}

\section{Keywords}

Abstract, Introduction, Basic Construction of Scaling Concepts Daubechies Wavelets, Wavelet Transformation, Results and Discussion, Error Metrics, Conclusion, References.

\section{INTRODUCTION}

A scaling or Dilation is a transformation which produces the resultant image that is the same shape as the given image. But the size is different. It expands or shrinks the original picture. The description of scaling includes the scale factor or ratio and the center of the scaling. The centre is a fixed point in the plane about which all the points are stretched or contracted. It is invariant point.

For two dimensional pictures, if there are $\mathrm{x}$ and $\mathrm{y}$ coordinates it can be written as

$$
S_{k}(x, y)=(k x, k y)
$$

If the scale factor, $\mathrm{k}$ is more than 1 , the output image is the enlargement of the original image. If the scale factor, $\mathrm{k}$ is less than 1 , the output image is the reduction of the original image .Congruent figures are obtained when it is equal to 1.[2]. The midpoint remains the same in both the figures. This scaling factor is a continuous factor. For the discrete basis $\mathrm{k}=2^{\mathrm{n}}$ where $\mathrm{n}$ is an integer. Thus the function can be rewritten as

$$
s_{n(x, y)}=\left(2^{n} x, 2^{n} y\right)
$$

Once the support of the above function becomes narrower, its frequency is higher. We can get approximate high frequency value by the higher order scaling. The compression of an image can be achieved by transforming the data, projecting it on a basis of functions and then this transform is encoded. But this transform must accept nonstationarity and be well localized both in space and frequency according to the nature of image and signal and the mechanisms of human vision.

A wavelet[3] has wave like form structure with an amplitude that begins with zero, increases, then it decreases back to zero level. That is, It is a Mathematical function used to divide the given function or continuous time function into different scale values.

A frequency range value is assigned to each scale component and it can be studied with a resolution that matches its scale.A wavelet transform which is a representation of a function of the wavelets that has scaled and translated copies of a finite wavelength or fast decaying oscillating waveform.

\section{BASIC CONSTRUCTION OF SCALING CONCEPTS}

To explain about the scaling function consider a continuous function[1]

$$
\boldsymbol{s}(\boldsymbol{t})=\left\{\begin{array}{l}
\mathbf{0}, \mathbf{0} \leq \boldsymbol{t}<1 \\
\mathbf{1}, \text { otherwise }
\end{array}\right.
$$

The scaled version of the above is defined as $S_{k}(t)$ such that

$$
\boldsymbol{s}_{\boldsymbol{k}}(\boldsymbol{t})=s(k t)
$$

scaling factor $(\mathrm{k})$ is a continuous factor. As $\mathrm{k}$ value is more, the support of this scaled basis will become narrower. For the discrete basis $\mathrm{k}=2^{\mathrm{n}}$ where $\mathrm{n}$ is an integer. Thus the above function can be rewritten as

$$
\boldsymbol{s}_{\boldsymbol{n}}(\boldsymbol{t})=s\left(2^{n} t\right)
$$

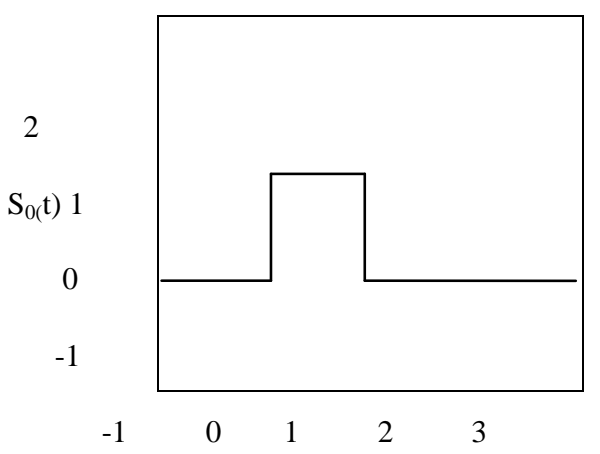

$(\mathrm{t})$

Fig.1 $\boldsymbol{s}_{\boldsymbol{n}}(\boldsymbol{t})=s_{0}(t)$

The above figures shows the scaling operations based on time and $S_{n}(t)$ where $n$ may have both positive and negative values.[5] 


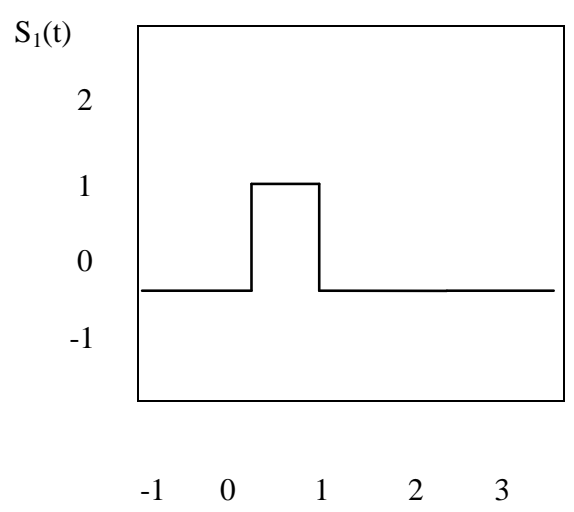

(t)

Fig.2 $\boldsymbol{s}_{\boldsymbol{n}}(\boldsymbol{t})=s_{1}(t)$

The above two functions $\mathrm{s}_{0}(\mathrm{t}), \mathrm{s}_{1}(\mathrm{t})$ are not orthogonal[4] to each other (orthogonality means the original signal is split into a low and high frequency part and enabling the splitting without duplicating the informations).because

$$
\begin{aligned}
s_{0}(t) s_{1}(t)> & =\int_{-\infty}^{\infty} s_{0}(t) s_{1}(t) d t \\
& =\int_{0}^{1 / 2} d t \\
& =1 / 2
\end{aligned}
$$

But if we apply Gram-Schmidt process to obtain a set of orthonormal basis from existing $\left\{\mathrm{s}_{\mathrm{n}}(\mathrm{t})\right\}$.

The procedure is

$$
\begin{array}{r}
\boldsymbol{s}_{\boldsymbol{n}}(\boldsymbol{t})=s_{0}(t)=s(t) \\
s_{1}(t)=s_{1}(t)-\frac{<s_{0}(t) s_{1}(t)>}{<s_{0}(t) s_{1}(t)>} s_{0}(t) \\
=\quad \begin{aligned}
\frac{1}{2} \quad & 0 \leq t<\frac{1}{2} \\
& -\frac{1}{2} \quad \frac{1}{2} \leq t<0
\end{aligned} \\
0 \text { otherwise } \\
=\frac{w(t)}{2} \text { (wavelet equation) }
\end{array}
$$

After applying continuously this process it is inspected that $\mathrm{s}(\mathrm{t})$ has a nonzero mean value while $\mathrm{w}(\mathrm{t})$ is zero in average. The $s(t)$ has 2 jumps at $x=0,1$ while $w(t)$ jumps at $x=0,1 / 2,1$. Therefore the power of $\mathrm{s}(\mathrm{t})$ is more compact at low frequencies while the power of $\mathrm{w}(\mathrm{t})$ concentrates at relatively high frequencies. In formal $s(t)$ is called scaling function to do approximation and $w(t)$ is called wavelet function to find the details. According to scaling concepts the angles, Parallelism(Parallel lines remain parallel) Orientation(lettering order remains the same), Colinearity (points stay on the same lines) are not changed in both the original and resultant figures. It is maintained in each figure. But the Distance is not preserved (i.e) the figures are not isometric.

Conceptually, the scaling function is the low frequency component of the previous scaling function in 2 dimensions. So there are one 2D scaling finction. But the wavelet function is related to the order to apply the filters If it is separable. If both scaling function and wavelet functions are separable the summation can be decomposed into two stages. Initially along the $\mathrm{x}$-axis and about the $\mathrm{y}$ axis. The fast wavelet transform is applied to accelerate the speed.

This section can be concluded that the scaled version wavelets allow us to analyze the picture in different scale.

\section{HAAR WAVELETS}

This Model is based on 'haar' Wavelet transformation with decomposition level 2.Haar Wavelet[5] is the simplest wavelet in the wavelet family. It has sequence of rescaled square-shaped functions. In the field of image compression analysis wavelets transforms have widely used. There are various wavelet transforms are used in the area of image compression and decompression. EZW,SPHIT,WDR, ASWDR. Among all these scaling and wavelet based image compression scheme ASWDR provides greater compression ratio. But SPHIT is superior in the quality of image and it provides higher PSNR than DWT. 2D wavelets are often used for image compression.

\section{DAUBECHIES WAVELETS}

The Daubechies wavelets[6] are the orthogonal wavelets (i.e) The inverse transform is adjoint of the original wavelet transform. It is defined in terms of resulting scaling and wavelet functions. The scaling function is a refinable equation that is a fractal functional equation which can be called as refinement equation or twin scale equation or dilation equation

$$
s(x)=\sum_{k=0}^{n-1} a_{k} s(2 x-k)
$$

Where the sequence $\left(a_{0} \ldots . . a_{n-1}\right)$ of real numbers is called a scaling sequence or scaling mask.

The wavelet equation may also have similar linear combination as follows:

$$
w(x)=\sum_{k=0}^{m-1} b_{k} s(2 x-k)
$$

Where the sequence $\left(b_{0} \ldots \ldots b_{m-1}\right)$ of real numbers is called a wavelet sequence or wavelet mask.

An important condition to be followed for the orthogonality of the wavelets is that the scaling sequence is orthogonal to any shifts of it by an even number of coefficients.

$$
\sum\left(a_{n} a_{n}\right)+2 m=2 \delta_{m}, 0 \text { where } n € z
$$

In this case , $\mathrm{M}=\mathrm{N}$ of coefficients are same in number both in scaling and wavelet sequence. The wavelet sequence can be calculated as $b_{n}=(-1) a_{n-1-n} \cdot n$ is the wavelet index.(i.e) 2 for D2 (Haar wavelet). $k$ is the coefficient index.

Wavelets[9] are mainly used for image compression and it uses smooth scaling and wavelet functions. Following JPEG2000, Daubechies 9/7 wavelets(i.e)Cohen DaubechiesFeaureau wavelets can be used for compression. 


\section{WAVELET TRANSFORMATIONS}

The Wavelet Transformations[7] divides the image into a set of sub images with different resolutions related to different frequency domains. The compression of an image can be achieved or based on the wavelet transform and a vector quantization coding scheme. The wavelet transformation uses JPEG 2000 standard. The performance of JPEG and JPEG2000 are the same at low compression ratio. But JPEG 2000 works better than that of JPEG at high compression ratio since it has no block effect. JPEG understands the whole image as a single unit and take 2D wavelet transformations in many levels. JPEG 2000 uses two different kinds of wavelet transformations [8] such as Biorthogonal Daubechies 5/3 for lossless compression and a Daubechies $9 / 7$ for lossy compression. Since Daubechies $9 / 7$ has quantization noise that depends on the precision of the decoder, it is an irreversible transform method. But according to Daubechies $5 / 3$ the coefficients are integers and there is no need to rounding of the results.

\section{RESULTS AND DISCUSSION}

The given image is compressed and decompressed using MATLAB source code. The compression ratio[10] and bits per pixels are calculated and compared with JPEG standard. This Model provides better results than JPEG standard for higher compression ratios. However there are no blocking effects in reconstructed image by wavelet based method.

The Matlab source Code supports both gray scaled and RGB images.

The algorithm of the source code used is:

1.Reading an image.

2.Converting into grayscale image if it is RGB

3. Decomposition of images using wavelet transformation for the level $\mathrm{N}$.

4.Selecting and assigning a wavelet transformation for image compression.

5.perform image compression

6.Decompress the image based on wavelet decomposition level

7.Plot the original image, compressed, reconstructed image

There are two interfaces in MatLab[11] which can be used for compressing the images and they are command line and GUI. Both the interfaces are involved in changing the image into given decomposed level with a specified wavelet.



Fig.3 original, compressed and reconstructed cameraman,testpat1,rice, lena_gray,peppergray,walkbridge,shot1,bacteria output images using MATLAB with scaling level 2

\section{ERROR METRICS}

There are two types of error measurements[12] to compare the different types of image compression techniques. They are Mean Square error and the Peak signal to Noise Ratio. The MSE is the cumulative squared error whereas PSNR is a measure of peak error between the compressed image and original image.

$$
\begin{gathered}
\text { Error }(E)=\text { original }- \text { Reconstructed image } \\
M S E=E /(\text { Image size }) \\
P S N R=20 * \log _{10}\left(\frac{255}{\sqrt{M S E}}\right) \\
S N R=20 * \log _{10}\left(\frac{p_{\text {signal }}}{p_{\text {noise }}}\right)
\end{gathered}
$$

If the error is minimum, MSE value will be less and it translates to a high value of PSNR.PSNR value should be higher for the better compression. The 'signal' is the original image and Noise is the error in the reconstructed image. Thus a compressed image[12] is a better image if it has lower MSE and higher PSNR.SNR value is the Ratio between the meaningful information and the unwanted information. It is a measure of the signal strength related to background noise.

Table.1 shows CR(compression ratio),MSE(Mean squared error),PSNR(Peak signal to noise ratio),SNR(Signal to noise ratio) for the test images

\begin{tabular}{|l|l|l|l|l|}
\hline $\begin{array}{c}\text { Test } \\
\text { Images }\end{array}$ & CR & MSE & PSNR & SNR \\
\hline $\begin{array}{l}\text { Camera } \\
\text { man }\end{array}$ & 84.5505 & 36.7151 & 32.5164 & 13.4368 \\
\hline rice & 84.5474 & 20.3195 & 35.0857 & 13.4795 \\
\hline lena & 84.5566 & 38.5866 & 32.3004 & 13.46 \\
\hline Testpat1 & 84.5459 & 176.958 & 25.6861 & 13.2465 \\
\hline Peppers & 84.6184 & 3.9035 & 12.2503 & 13.5748 \\
\hline $\begin{array}{l}\text { Walk } \\
\text { bridge }\end{array}$ & 84.5623 & 2.9183 & 13.5135 & 13.6836 \\
\hline Shot1 & 84.0443 & 3.1101 & 12.9805 & 13.4589 \\
\hline bacteria & 84.2827 & 3.0986 & 12.9765 & 13.3365 \\
\hline
\end{tabular}




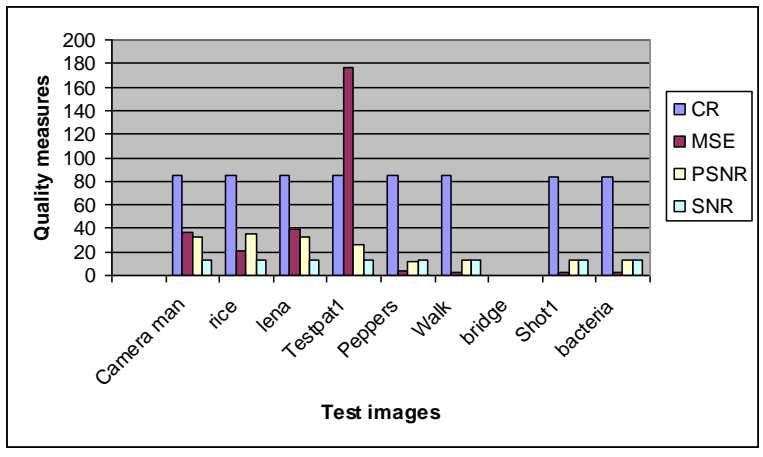

Chart1:Compression Ratio,(CR) Mean squared error,(MSE) Peak signal to Noise Ratio(PSNR), SNR(Signal to Noise Ratio) for the images cameraman, rice,lena testpat1,peppergray and walkbridge,shot1,bacteria.

\section{CONCLUSION}

Modern cameras have the resolutions with the range of tens of megapixels and these images need to compress before storage and transfer. The HAAR Transformation[13] is used to transfer the image into a matrix in which each element of the matrix represents a pixel. That is, a $128 * 128$ matrix is saved for a $128 * 128$ image. But JPEG standard cuts the original image into $8 * 8$ sub images and each sub image is an $8 * 8$ matrix. In this paper, different test images are compressed and decompressed using 'HAAR' wavelet transformation. The effects of different scaling[14] and wavelet functions were applied and implemented in various test images. The filter orders, number of decompositions, Image contents, and compression ratios, different quality measures such as MSE, PSNR, and SNR were also calculated for the above test images and the results were examined.

\section{ACKNOWLEDGEMENTS}

I would like to express my gratitude to my guide Dr.Rama for her intellectual guidance and support.

I express my thanks to my family members for their constant support

I wish to express my thanks to the lab technicians Ms.Ramya and Mr saravanan for their assistance and help.

\section{REFERENCES}

[1] http://disp.ee.ntu.edu.tw/tutorial/WaveletTutorial.pdf.
[2] IEEE Transactions on image processing, Vol.12, NO.12, December 2003, 1449. Non linear wavelet transforms for image coding via lifting Roger L.Claypoole, Jr., Senior Member, IEEE, Geoffrey M.Davis, Wim Sweldens, Member, IEEE, and Richard G. Baraniuk, Fellow, IEEE.

[3] Adative Wavelet rendering by Ryan S. Overbeck Columbia University, Craig Donnery Columbia University. Z. Ravi Ramamoorthi, University of California, Berkeley.

[4] http://en.wikipedia.org/wiki/Orthogonalwavelet.

[5] ACEEE Int.J. on information Technology,Vol.01.No.02,sep2011, Image Compression using WDR \& ASWDR Techniques with different wavelet Codecs By S.P.Raja, Dr.A.Suruliandi, Manonmaniam sundaranar University.

[6] http://en.wikipedia.org/wiki/daubechies wavelet

[7] K. Ramachandran, S.LoPresto and M.Orchard," Image coding based on mixture modeling of wavelet coefficients and a fast estimation quantization framework" in Proc. Data compression Conf., Snowbird, UT, March 1997.

[8] I. Daubechies and W.Swwldens, "Factoring wavelet transforms into lifting steps, "J.Fourier Anal. Appl.. Vol 4, no 3,PP-245-267 1998.

[9] R.Calderbank, I. Daubechies, W. Sweldens and B.L.Yeo, "Wavelet transform that map integers to integers" Appl Comput,Harmon Anal.,vol 5, No 3,,pp332-369,1998.

[10] Walker.J.S.A Primer on wavelets and their Scientific Applications Boca Raton, Fla Chapman \& Hall/CRC, 1999.

[11] Robi Polikar, " the wavelet tutorial. http:// engineering.rowan.edu/ polikar/wavelets/wttutorial.html.

[12] Misiti. M. Misiti,Y. Oppenheim, G.Pggi, J-M. wavelet Toolbox Users Guide, Version 2.1.The Mathworks, Inc 2000.

[13] Hubbard, Barbara Burke. he World according to wavelets A.K Peters Ltd, 1995.

[14] C.Gargour M Gabrea V Ramachandran J M Lina," A short Introduction to wavelets and their applications", Circuits and systems Magazine, IEEE, Vol.9, No.2 (05 June 2009), PP.57-68,[2]. 